\title{
Single and double polarization asymmetries from deeply virtual exclusive $\pi^{0}$ electroproduction
}

\author{
Andrey KIM* \\ University of Connecticut \\ E-mail: kenjo@jlab.org \\ Harut Avakian, Volker Burkert et al. (CLAS collaboration) \\ Jefferson Lab
}

\begin{abstract}
The target and double spin asymmetry measurements of exclusive $\pi^{0}$ electroproduction were performed for the first time in DIS region at Jefferson Lab using the CEBAF Large Acceptance Spectrometer (CLAS) and longitudinally polarized proton target. The wide kinematic coverage and good resolution of CLAS allowed measurements in full azimuthal range providing an opportunity to extract single and double spin asymmetries proportional to polarized structure functions. Their angular dependencies in hadronic center-of-mass system were analyzed, and extracted moments are compared to recent theoretical handbag-based calculations based on chiral-even and chiral-odd GPDs contributions. The interpretation of present results within the framework of the modified perturbative approach and their use as a constraint for models of the $t$ behavior will be discussed.
\end{abstract}

XXII. International Workshop on Deep-Inelastic Scattering and Related Subjects, 28 April - 2 May 2014

Warsaw, Poland

${ }^{*}$ Speaker. 


\section{INTRODUCTION}

In recent years, parton distribution functions have been generalized to contain information not only on the longitudinal but also on the transverse distributions of partons in a fast moving hadron. The Generalized Parton Distributions (GPDs) [1, 2, 3] add important piece of information that is missing in one-dimensional parton densities, in particular, distribution of partons in the plane transverse to the direction of motion. They are closely related to the nucleon electromagnetic form factors as well and contain information that cannot be accessed by either of these quantities. The most important aspect is that GPDs can provide detailed knowledge about the space-momentum distributions of quarks and gluons within the nucleon and about the contribution of quark orbital angular momentum to the nucleon spin. GPDs contain the information needed to construct a multidimensional image of the internal structure of the nucleon.

Exclusive electroproduction processes $\gamma^{*}\left(Q^{2}\right)+N \rightarrow M+N$ (N=nucleon, $M=\gamma$, meson) offer a unique opportunity to study the structure of the nucleon as one varies both the size of the probe - the photon virtuality, $Q^{2}$ - and the momentum transfer to the nucleon, $t$. It has been argued that exclusive productions of mesons [4] effectively proceeds via a partonic mechanism, and can be described in terms of the GPDs in the nucleon. This opens the prospect of using exclusive meson electroproduction processes as a means to systematically explore the quark structure of the nucleon.

The differential cross section for exclusive meson electroproduction off an longitudinally polarized proton target is given by [5]:

$$
\begin{aligned}
\frac{2 \pi}{\Gamma\left(Q^{2}, x_{B}, E\right)} \frac{d^{4} \sigma}{d Q^{2} d x_{B} d t d \phi_{\pi}}=\quad & \sigma_{T}+\varepsilon \sigma_{L}+\varepsilon \sigma_{T T} \cos 2 \phi+\sqrt{2 \varepsilon(1+\varepsilon)} \sigma_{L T} \cos \phi \\
& +P_{b} \sqrt{2 \varepsilon(1-\varepsilon)} \sigma_{L T^{\prime}} \sin \phi \\
& +P_{t}\left(\sqrt{2 \varepsilon(1+\varepsilon)} \sigma_{U L}^{\sin \phi} \sin \phi+\varepsilon \sigma_{U L}^{\sin 2 \phi} \sin 2 \phi\right) \\
& +P_{b} P_{t}\left(\sqrt{1-\varepsilon^{2}} \sigma_{L L}+\sqrt{\varepsilon(1-\varepsilon)} \sigma_{L L}^{\cos \phi} \cos \phi\right)
\end{aligned}
$$

where $\mathbf{P}_{\mathbf{b}}$ is the beam polarization, $\mathbf{P}_{\mathbf{t}}$ is the target polarization, $\varepsilon$ is virtual photon polarization, $\phi$ is an azimuthal angle between the electron scattering plane and the hadronic reaction plane, $\sigma_{\mathbf{T}}$, $\sigma_{\mathbf{L}}, \sigma_{\mathbf{T T}}, \sigma_{\mathbf{L T}}, \sigma_{\mathbf{L T}}, \sigma_{\mathbf{U L}}^{\sin \phi}, \sigma_{\mathbf{U L}}^{\sin 2 \phi}, \sigma_{\mathbf{L L}}, \sigma_{\mathbf{L L}}^{\cos \phi}$ are the structure functions depending on $Q^{2}, x_{B}, t$.

Within a standard collinear factorization scheme it was initially proposed that factorization in DVMP works rigorously for longitudinal virtual photons. However, factorization for transverse polarization case has in fact not been disproven although a dedicated proof is missing. Estimates for electroproduction of pseudoscalar mesons have been performed at small values of skewness within the handbag approach which is based on factorization in hard parton subprocesses and soft generalized parton distributions (GPDs) [6, 7, 8] (see Fig.1) under the assumption that a form of factorization working for both longitudinal and transverse virtual photons.

Pseudoscalar meson electroproduction, particularly $\pi^{0}$ production in the reaction $e p \rightarrow e^{\prime} p^{\prime} \pi^{0}$, was identified as especially sensitive to the parton helicity-flip subprocesses. While the interpretation of $\pi^{+}$production is complicated by the dominance of the longitudinal $\pi^{+}$-pole term, the $\pi^{0}$ production, where that contribution is absent, may become a unique source of information on transversity GPDs [7, 8]. During the past few years, two parallel approaches have been developed 


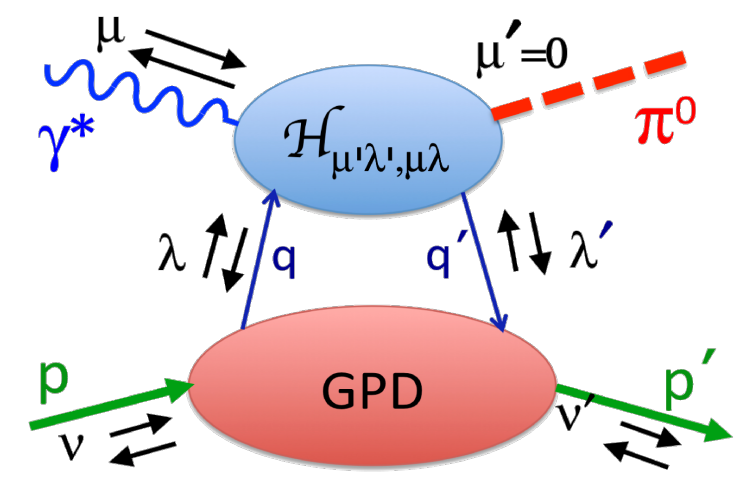

Figure 1: Schematic $\pi^{0}$ electroproduction in the handbag mechanism framework. The helicities of initial (final) nucleon and quark are denoted as $v\left(v^{\prime}\right)$ and $\lambda\left(\lambda^{\prime}\right)$ respectively. The incident virtual photon and produced $\pi^{0}$ meson have $\mu$ and $\mu^{\prime}$ helicities.

utilizing chiral odd GPDs in the calculation of pseudoscalar electroproduction: the GoloskokovKroll (GK) model[9, 7, 8] and Goldstein-Liuti (GL) model[10] of GPDs parameterization. Although different in details they both lead to sizable transverse photon amplitudes, as evidenced in the CLAS data. Inclusion of the chirally-odd twist-3 components of the hard exclusive amplitude gives results in fair agreement with the measured cross sections [11]. A combination of $\widetilde{H}_{T}$ and $E_{T}$ plays a particularly prominent role.

The unpolarized and polarized structure functions can be expressed as a combinations of the partonic subprocess amplitudes and GPDs convolutions, and measurements of beam, target and double spin asymmetries and extraction of azimuthal moments provide several observables, combined analysis of which may allow separation of contributions from different underlying GPDs.

\section{EXPERIMENT AND DATA ANALYSIS}

To study an exclusive reactions the dedicated experiments were carried out in Hall B at Jefferson Lab. The "eg1-dvcs" experiment was one of the first experimental studies of DV $\pi^{0} \mathrm{P}$ with a polarized target. Longitudinally polarized high energy electron beam provided by Thomas Jefferson National Accelerator Facility strikes the longitudinally polarized proton in frozen ammonia, and the outgoing particles in the reaction are detected using the CEBAF Large Acceptance Spectrometer and newly installed Inner Calorimeter. In the eg1dvcs experiment the data were collected between February and September 2009. The typical beam current was around $7 \mathrm{nA}$. The integrated luminosity was approximately $75 \mathrm{fb}^{-1}$. The beam polarization was measured using Moller polarimeter with an average polarization of $85 \%$. The target polarization was monitored in real-time using Nuclear Magnetic Resonance (NMR) system. The average proton polarization for $\mathrm{NH}_{3}$ target was $78 \%$ and $74 \%$ for positively and negatively polarized targets, respectively. Additionally the empty and Carbon solid targets were used for the nuclear background study purposes.

The event selection criteria required identification of electron, proton and at least two photons. The cuts on kinematic variables were placed to ensure that the event is in DIS region: $Q^{2}>1 \mathrm{GeV}^{2}$, $W>2 \mathrm{GeV}$. The electron was identified by the appropriate energy deposition in electromagnetic 


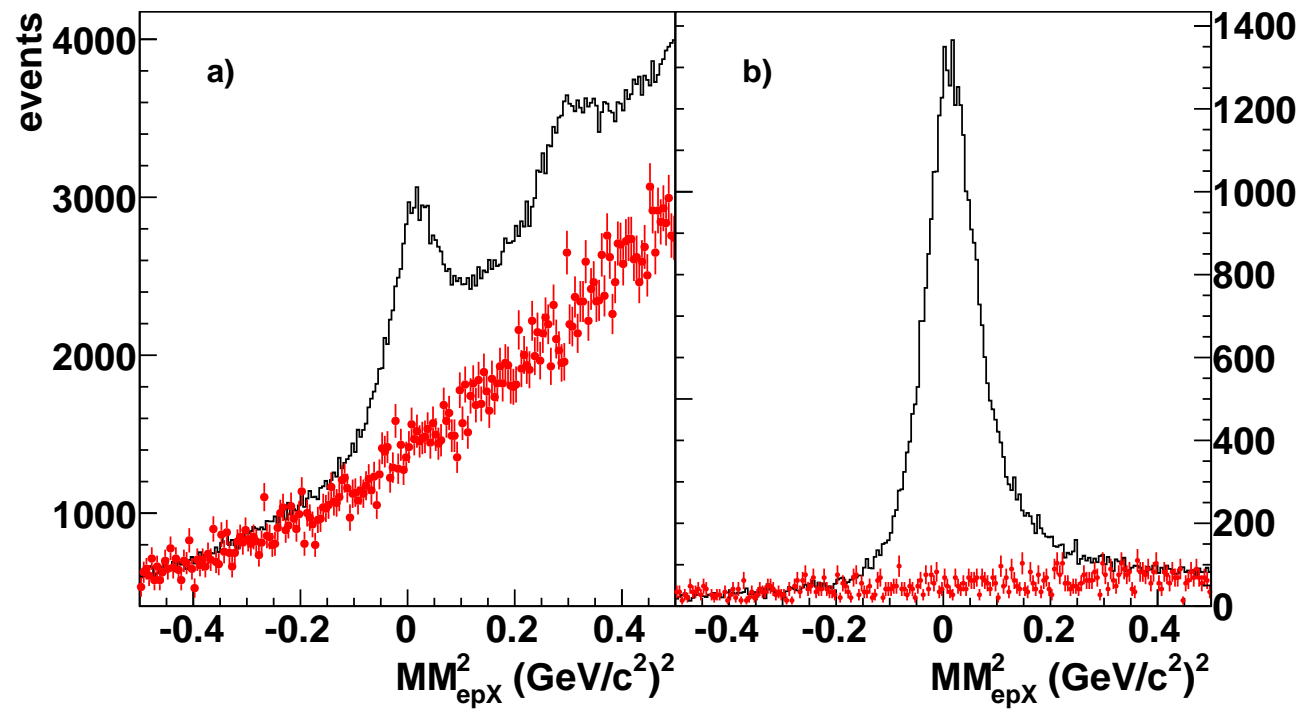

Figure 2: Distributions of missing mass squared of (ep) system for the reaction $e \vec{p} \rightarrow e^{\prime} p^{\prime} \pi^{0}$ before $(a)$ and after $(b)$ exclusivity cuts to other variables are applied. The ${ }^{12} \mathrm{C}$ data (markers) are normalized to the ${ }^{14} \mathrm{NH}_{3}$ data (line) using the negative tail of $M M_{e p X}^{2}$.

calorimeter, and proton was identified by the time-of-flight from the target to the CLAS scintillator counters.

Two detector systems were used in CLAS to identify the photons from $\pi^{0}$ decay over wide momentum and angular range. The Inner Calorimeter (IC), installed downstream of the target, provided detection of photons at low polar angle, and Electromagnetic Calorimeter (EC) provided photons detection at higher polar angle. The $\pm 3 \sigma$ cuts on invariant mass of photons pair was placed to identify the $\pi^{0}$ event. The remaining background was accounted using the side-band subtraction method for each kinematic bin and beam/target helicity state individually.

Detection of all four final-state particles allowed us to select the exclusive $\pi^{0}$ events on polarized proton despite the prevalent number of events on unpolarized proton from nitrogen through the development of exclusivity cuts on variables, such as missing energy $\left|E_{\text {miss }}\right|$, missing mass squared $M M_{e p X}^{2}$ and cone angle between reconstructed and predicted pions $\theta_{x \pi^{0}}$. Their effectiveness of nuclear background suppression is shown on Fig.2.

To take into account the remaining nuclear background from unpolarized nitrogen the dilution factor was used. It was measured using the data collected from carbon target that was chosen to mimic the contribution from nitrogen. In order to estimate this contamination the data from carbon runs were analyzed in the same kinematic region, and dilution factor was calculated:

$$
f=1-\frac{s N_{C}}{N_{A}}
$$

where $N_{C}\left(N_{A}\right)$ are the luminosity-normalized events from integrated carbon and ammonia runs, and $s$ is a scaling factor that accounts for the difference between carbon and nitrogen. The strict exclusivity cuts provided us a mechanism that allowed to separate the polarized proton events from unpolarized nuclear background suppressing the latter below $15 \%$. 
The $e p \rightarrow e p \pi^{0}$ spin asymmetries depend on virtual photon's squared four-momentum $Q^{2}$, Bjorken scaling variable $x_{B}$, squared 4-momentum transfer $t$ to the proton and on angle $\phi$ between the leptonic and hadronic planes. The experimental data were divided into two bins in $Q^{2}, x_{B}$ plane, five bins in $-t$ and eleven bins in $\phi$, and target and double spin asymmetries were measured in each kinematic bin over the wide range of $\left\{Q^{2}, x_{B}, t, \phi\right\}$ space.

The azimuthal dependencies of all three experimental observables (beam, target and double spin asymmetries) were fitted together using the chi-square minimization to take advantage of the same denominator. The asymmetry moments proportional to the ratios of structure functions are extracted as fit parameters for each kinematic bin and shown on Fig.3.
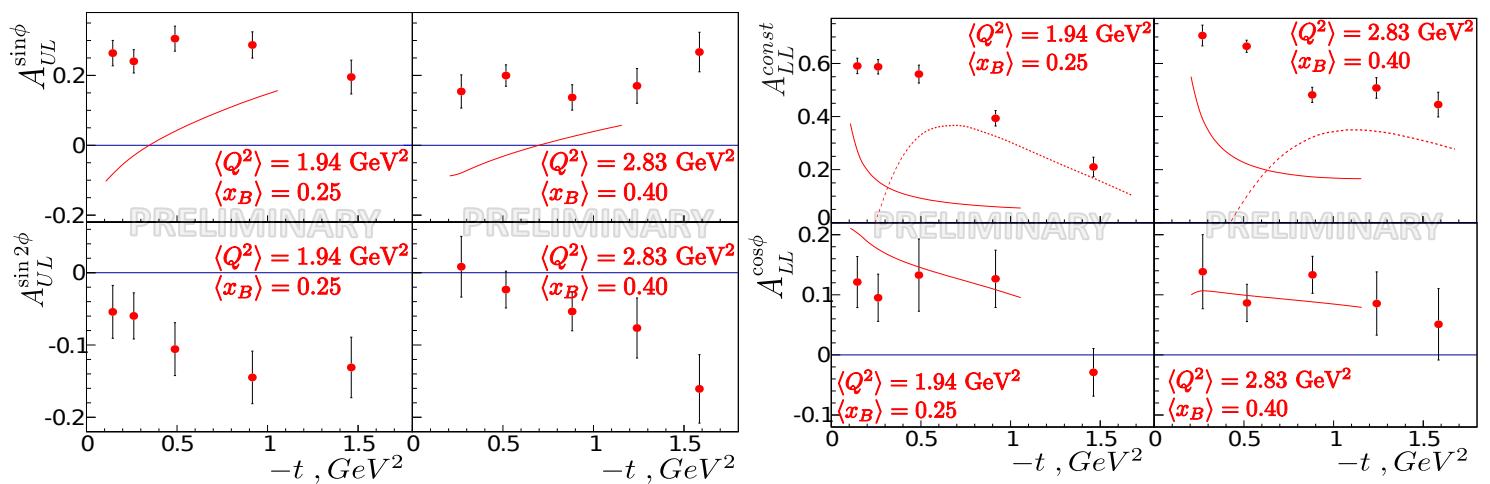

Figure 3: Target (left) and double (right) spin asymmetries moments for two kinematic bins in $Q^{2}, x_{B}$. The solid and dashed curves represent theoretical predictions from the model of Ref. [8] and [10].

\section{SUMMARY}

The longitudinally polarized beam and longitudinally polarized proton target in eg $1 \mathrm{dvcs}$ experiment allowed measurements of three polarization observables for deeply virtual exclusive $\pi^{0}$ electroproduction in wide kinematic range at CLAS. The target and double spin asymmetries were measured for the first time in DIS region.

The first interpretation of newly obtained data from Goloskokov-Kroll and Goldstein-Liuti models perspective was done. The contributions from transverse photons are dominant in the $\pi^{0}$ production. The behavior of the asymmetries as a functions of $t$ is defined by relation between two primarily contributing GPDs, $H_{T}$ and $\bar{E}_{T}$. The calculations from GK model shows the dominance of $\bar{E}_{T}$ over $H_{T}$ and agree rather well with recent CLAS measurement of unpolarized cross sections of $\pi^{0}$ electroproduction [11]. However, the correct parameterization of non-dominant $H_{T}$ is required to describe the polarized observables. It is clear from data and model disagreement that the relative $H_{T}$ contribution is underestimated.

The double spin asymmetries $A_{L L}^{\text {const }}$ were compared to the Goldstein-Liuti model prediction. The more extensive data-driven determination (from EM form factors, PDFs, DVCS and some lattice gauge parameters) of the chiral-even helicity amplitudes and GPDs leads to better agreement with measured observables especially in the high- $t$ region. The interplay between $\bar{E}_{T}$ and $H_{T}$ GPDs as well as $\tilde{E}_{T}$ defines the model behavior. The calculations from GL model agree with our 
experimental results better at high- $t$ values, where $E_{T}$ dominates . However, at low- $t$ region the parameterizations of $H_{T}$ and $\tilde{E}_{T}$ and their contributions relative to $\bar{E}_{T}$ seem to be underestimated.

Discrepancies between data and model calculations require further clarifications. Unfortunately, the precise calculation of all details of $\pi^{0}$ electroproduction is not possible at present. The current model predictions of single and double spin asymmetries do not agree with eg1dvcs measurements due to the lack of data to constrain the parameterizations of transverse GPDs and their relative phases. The exact treatment of the twist- 3 contribution is also uncertain.

The contribution of transverse photons is decreasing as $Q^{2}$ grows. The Jefferson Lab upgrade program may provide data at the region where twist- 2 contributions are dominant. It is not yet clear what value of $Q^{2}$ is required. However, future measurements of polarized observables will supply further constraints for GPDs and may test model independent features of the reaction mechanism.

\section{References}

[1] X-D. Ji, Gauge invariant decomposition of nucleon spin, Phys. Rev. Lett. 78 (610) [hep-ph/9603249].

[2] A.V. Radyushkin, Scaling Limit of Deeply Virtual Compton Scattering, Phys. Lett. B 380 (417) [hep-ph/9604317].

[3] A.V. Belitsky, D. Mueller, A. Kirchner, Theory of deeply virtual Compton scattering on the nucleon, Nucl.Phys B629 (323) [hep-ph/ 0112108$].$

[4] J.C. Collins, L. Frankfurt, M. Strikman, in proceedings of Madrid Workshop on Low x Physics, 296.

[5] M. Diehl, A. Sapeta, On the analysis of lepton scattering on longitudinally or transversely polarized protons, Eur. Phys. J. C41 (515) [hep-ph/ 0503023$].$

[6] S. Ahmad, G.R. Goldstein, S. Liuti, Nucleon Tensor Charge from Exclusive $\pi^{0}$ Electroproduction, Phys. Rev. D79 (054014) [hep-ph/ 0805 . 3568].

[7] S.V. Goloskokov, P. Kroll, An attempt to understand exclusive pi+ electroproduction, Eur. Phys. J. C65 (137) [hep-ph/0906.0460].

[8] S.V. Goloskokov, P. Kroll, Transversity in hard exclusive electroproduction of pseudoscalar mesons, Eur.Phys.J. A47 (112) [hep-ph/1106.4897].

[9] S.V. Goloskokov, P. Kroll, The role of the quark and gluon GPDs in hard vector-meson electroproduction, Eur.Phys.J. C53 (367) [hep-ph/ 0708 .3569].

[10] G.R. Goldstein, J.O. Hernandez Gonzalez, S. Liuti, Flexible Parametrization of Generalized Parton Distributions: The Chiral-Odd Sector, to be published, hep-ph/1311.0483

[11] I. Bedlinskiy et al. (CLAS Collaboration), Measurement of Exclusive $\pi^{0}$ Electroproduction Structure Functions and their Relationship to Transverse Generalized Parton Distributions, Phys. Rev. Lett. 109 (11) 112001 [hep-ph/1206.6355] 